\title{
Vertrauen, religionssoziologisch
}

Die Soziologie beginnt misstrauisch: Soziale Ordnung wird zwar für durchaus möglich aber unwahrscheinlich gehalten, weshalb Soziologen sich dafür interessieren, wie es trotzdem dazu kommt. Die mit sozialen Angelegenheiten einhergehende Ungewissheit setzt bereits bei elementaren Interaktionen ein: Für die Beteiligten an einer Interaktion sind ihre nächsten Entscheidungen und Handlungen gegenseitig nur sehr begrenzt vorherseh- oder steuerbar, es sind jeweils nicht determinierte Reaktionen auf den vorhergehenden Schritt des Gegenübers. Vieles ist möglich, weniges ist notwendig und das auf beiden Seiten, weshalb sich die Situation Talcott Parsons (1964 [1951]: 10-11) folgend als «doppelte Kontingenz» bezeichnen lässt.

Soziologisch wird nach Strukturen Ausschau gehalten, mit der diese Ausgangslage für die Beteiligten wie für den Beobachter festere, verlässlichere und verstehbarere Formen annimmt. Beispiele für solche Strukturen ist das Ködern mit positiven Sanktionen wie z.B. Geld, oder machtvolle Drohungen mit negativen Sanktionen wie z.B. physischer Gewalt. Eine ganz andere Möglichkeit der Reduktion von Komplexität ist Vertrauen: Im generalisierenden Rückgriff auf eine Einschätzung des Gegenübers und ohne auf spezifische Informationen über dessen Verhalten in der problematischen Situation zurückzugreifen oder sich durch Sanktionen abzusichern, traut man diesem Gegenüber etwas mehr oder weniger Spezifisches zu.

Der Begriff des Vertrauens findet sich bei den meisten prominenteren Soziologen, dies jedoch ohne einen zentralen Stellenwert einzunehmen. In der Religionssoziologie verschwindet «Vertrauen» fast ganz. Sollte sich das ändern? Im Folgenden soll diese Frage beantwortet werden und dies nicht über die Sammlung und Sortierung sämtlicher religionssoziologischer Anwendungen von «Vertrauen», sondern über ein Ausloten von Möglichkeiten und Leistungen des Begriffes an zwei Polen des religionssoziologischen Gegenstandbereichs: Auf der einen Seite Religion, die durch Rituale geprägt ist, und auf der anderen Seite Religion, die sich der meisten Rituale entledigt und Glaubensüberzeugungen in ihrem Zentrum stehen hat. 


\section{Glaube}

Glaube allein ist ein religionssoziologisch wenig interessanter $\mathrm{Zu}$ stand, wenn nicht danach gefragt wird, was für Konsequenzen er für das Handeln zeitigt. Max Webers Ausführungen zum asketischen Protestantismus und dem modernen Kapitalismus bilden die klassische Auseinandersetzung mit dieser Frage. Hier soll sie mit der von Weber nur sehr spärlich verwendeten Vertrauensbegrifflichkeit betrachtet werden.

«Religiöse Bewußtseinsinhalte» hätten «auf die Lebensführung, die Kultur und die Volkscharaktere» in den von Weber betrachteten Kontexten des 17. Jahrhunderts eine Bedeutung gehabt, wie sie sich der «moderne Mensch» nur schwer vorstellen könne (vgl. Weber 1988 [1904]: 205). Weber interessiert sich für die Konsequenzen dieser Bewusstseinsinhalte und macht als dafür besonders bedeutsamen Fall die Calvinisten jener Zeit aus, die sich in ihrem vorherbestimmten aber unbekannten Heilszustand einem allmächtigen, allwissenden und unergründlichen Gott gegenüber sehen. In einer solchen Konzeption absentiert sich Gott aus einer durch doppelte Kontingenz geprägten Beziehung. Was er vorherbestimmt hat, lässt sich weder in Erfahrung bringen noch beeinflussen. Weder Zugehörigkeit noch Rituale haben Auswirkungen auf die göttlichen Entscheidungen, von welchen auch die bange Frage nach dem eigenen Heil bestimmt ist. Sämtliche magischen Mittel sind somit ausgeschaltet, die Religion ist entzaubert.

«Logisch» wäre zu erwarten gewesen, so Weber, dass die Calvinisten angesichts dieser Umstände in Fatalismus verfallen wären. Letztlich hätten Ungewissheit und der Mangel an Einfluss aber "psychologisch" dazu geführt, dass sie sich religiös umso mehr ins Zeug legten. Dies wurde dadurch ermöglicht, dass daran geglaubt wurde, dass es durchaus Anzeichen für den Heilszustand gäbe. Wer auserwählt ist, dem zeige sich das bereits im Diesseits, nämlich anhand der Bewährung in der gottgeschaffenen, weltlichen Ordnung. Als Indikatoren dafür galten rechtschaffenes Handeln, ethische Lebensführung und ein damit verbundener Erfolg im Berufsleben. Die durch diese Zeichen erzeugte Gewissheit lässt sich jedoch nicht kumulieren, vielmehr ist sie einer dauernden Bewährung unterworfen, wobei sich Ausrutscher nicht rituell-magisch ausbügeln lassen. Selbstvertrauen hinsichtlich des eigenen Gnadenstands lässt sich damit nur durch eine stetige Bewährung in der Lebensfuihrung, die durch einen Ethos von Rechtschaffenheit und Fleiss rational durchdrungenen war, herstellen und aufrecht erhalten. In Verbindung mit 
der andauernden Heilsunsicherheit führte also die Entzauberung der Welt zu einer hohen Spannung, die in ein andauerndes Selbstmisstrauen mündete, verstärkt durch die Unmöglichkeit, auf die kompensatorische Gnade oder erbetene Nachsicht eines Gottes zu vertrauen, der sich nicht auf Interaktion einlässt. Dieser Problematik konnte nur über ein fortlaufendes Aufrechterhalten von Selbstvertrauen durch Bestätigung im diesseitigen Leben begegnet werden.

Dies hatte gemäss Weber historische Konsequenzen, da eine solche Verlagerung des Vertrauens in ausserweltliche Akteure oder rituelle Handlungen auf die eigene Person und die daran anschliessende Rationalisierung der Lebensführung den Boden für eine bisher ungekannte Form wirtschaftlichen Handelns bereitete: den rationalen Kapitalismus.

\section{Rituale}

Die Unmöglichkeit ritualistischer oder anderweitiger Beeinflussung Gottes in diesen Formen des Protestantismus sah Max Weber als Konsequenz eines Prozesses der Entzauberung, womit er das zunehmende Verschwinden magischer Elemente aus der Religion bezeichnete und nicht etwa, wie oft unterstellt, eine generelle Säkularisierung. Als Kontrast zu dieser entzauberten, gilt es nun die verzauberte Religion zu betrachten. Auch sie soll zunächst als eine Situation der doppelten Kontingenz mit dem Menschen auf der einen Seite und einer übernatürlichen Macht auf der anderen erfasst werden. Zauber bzw. Magie bestehen dabei darin, dass der Mensch durch bestimmte Handlungen sein übernatürliches Gegenüber beeinflussen kann. Entscheidend ist dabei der Grad der Personalisierung des übernatürlichen Gegenübers: Dem Ethnologen James George Frazer (1963 [1922]) folgend, kann von einem Kontinuum zwischen einem stark personalisierten Gott hin zu Vorstellungen einer unpersönlichen Kraft ausgegangen werden. Ersterem wird höhere Kontingenz zugetraut: Gott lässt zwar mit sich reden und Bitten und Opfer lassen sich anbringen, er entscheidet jedoch letztlich selbstständig. Das führt dazu, dass Vertrauen in dieser Beziehung, in der gegenseitige Determination für unmöglich gehalten wird, wichtig ist.

Dem hier interessierenden Idealtyp einer «verzauberten Religion» kommt jedoch die zweite Konfiguration näher. Nicht Gott als Person, sondern eine unpersönliche Macht steht im Zentrum. Die Interaktion nimmt stark ritualisierte Formen an, das heisst diejenigen einer traditionalistischen, standardisierten und formalisierten 
Praxis. Die doppelte Kontingenz der Situation wird insofern stark eingeschränkt, als dass aus einer personalisierten Gottheit, die immer auch anders handeln könnte, eine Kraft, eine Gesetzmässigkeit wird, die es richtig zu beeinflussen gilt. Ist das Resultat nicht wie gewünscht, setzt also nach dem berühmten Regentanz kein Regen ein, liegt das einzig daran, dass das Ritual falsch durchgeführt wurde. Dem übernatürlichen Gegenüber wird kein Spielraum für Entscheidungen zugetraut, ihm kann weder vertraut noch misstraut werden. Dennoch ist Vertrauen im Spiel. Die rituelle Praxis stellt einen vertrauensbildenden Gegenwartsbezug her, man verlässt sich auf eine bestimmte Art koordinierter sozialer Interaktion. Bei Ritualen mit gemeinschaftlicher Beteiligung ist Vertrauen nicht etwas, was von einer Partei der anderen geschenkt wird, sondern rituelle Praxis wird zum Vollzug und zur Kommunikation gegenseitigen Vertrauens - umso mehr deshalb, weil Rituale keine Kapazität haben, um mit in ihrem Verlauf auftretenden Vertrauensbrüchen (klassisches Beispiel ist das Nein-Wort bei Trauungen) umzugehen. Rituale zelebrieren also gegenseitiges Vertrauen der Gemeinschaftsmitglieder: «tous sont accomplis dans un état de confiance» (Durkheim 1979 [1912]: 556). In der kollektiven Efferveszenz des Rituals werden nicht nur soziale Kategorien und gemeinschaftliche Symbole gestärkt, sondern auch das Vertrauen in die Gemeinschaft und ihre Mitglieder.

Zwei Kontrastierungen sind zu den vorangegangenen Ausführungen anzubringen, die beide Konsequenzen für die abschliessenden Überlegungen zum Vertrauensbegriff haben werden:

Erstens zeigen beide Beispiele, wie Religion im Handeln wirksam wird, entscheidend ist jedoch, was das Objekt und der Geltungsbereich des damit erzeugten Vertrauens ist. Bei Webers Calvinisten führt die Verabschiedung einer persönlichen übernatürlichen Macht aus der religiösen Interaktion zu einerVerlagerung des Vertrauens ins individuelle, innerweltliche Handeln. Im ritualistischen Beispiel wird auf eine ausserweltliche Praxis vertraut: Rituale sind durch Grenzziehungen gegenüber anderen Interaktionsformen gekennzeichnet und erzeugen damit einen Wirklichkeitsbereich mit eigenen Relevanzen und Regeln. Dies unterscheidet sich von der Durchdringung des Alltags durch einen religiösen Ethos, der den besagten Calvinisten zurVersicherung ihrer Erlöstheit diente.

Zweitens ist es erläuterungsreich, die weitere Argumentation Frazers anzuschauen und sich von ihm abzugrenzen: Magie sei letztlich ein kapitaler Irrtum, da dabei in gänzlich falsche Kenntnisse und unnütze Handlungen vertraut würde. Je stärker personalisiert das Gegenüber, desto verständlicher sei der Irrtum, da dabei immerhin 
das Ausbleiben des Regens göttlicher Willkür angelastet werden könne. Im Fall gänzlich verzauberter Religion, die Frazer als Zauber oder Magie bezeichnet, müssten jedoch die Regentänzer als sehr dumm oder zumindest äusserst verzweifelt gesehen werden. Obwohl ihre rituelle Praxis nichts tauge und damit ihre Vorstellungen stets widerlege, werde sie von den Unbelehrbaren wieder und wieder durchgeführt.

Die Kritik an Frazers Vorstellungen hielt sich nicht zurück: «Frazer wäre imstande $\mathrm{zu}$ glauben, dass ein Wilder aus Irrtum stirbt. (...) Frazer ist viel mehr savage, als die meisten seiner savages», lautet Ludwig Wittgensteins (1975: 44-45) harsche Kritik an Frazer, die sich aus soziologischer Perspektive wie folgt einordnen lässt: Frazer unterstellt der Praxis eine Logik, er sieht Rituale als Folgerungen aus einem System von geglaubten Kausalitätsverhältnissen, das die rituell Handelnden dazu anleitet, ihre Zwecke auf bestimmte Art und Weise zu verfolgen. Der viel einfachere Blick darauf, auf was tatsächlich vertraut wird, ist hier heilsam. Was in Ritualen zählt, ist die richtige Abfolge von Schritten und ihre kommunikative Kapazität beschränkt sich weitgehend darauf, mitzuteilen, was der nächste rituelle Schritt ist. Der Gegenstand des Vertrauens ist die Befolgung dieser Schritte, die Teilnehmer verlassen sich gerade in schwierigen Situationen darauf, dass es zu diesem Zeitpunkt richtig ist, so zu handeln. Der Glaube an eine Einbindung dieser Handlungsanleitungen in ein System von Gesetzmässigkeiten, die Ableitung ritueller Handlungen aus Glaubensmotiven, dürfte weitgehende Unterstellung sein. Aus ex post Rationalisierungen befragter Ritualteilnehmer können mit Frazer solche Verknüpfungen wohl hergestellt werden, jedoch sind Rituale typischerweise «traditionales Handeln» im Sinne Weber, es wird so gehandelt, weil nun einmal so gehandelt wird. Regentänze werden nicht praktiziert, weil man ihnen die Wetterbeeinflussung zutraut, sondern weil sie ein Vokabular für die Beschäftigung mit dem Wetter bereitstellen. Entsprechendes gilt für Horoskope, denen auch nicht aufgrund ihrer prognostischen Zuverlässigkeit vertraut wird. Auch die TV-Berichterstattung vor unwägbaren Sportwettkämpfen, in der von detaillierten Mutmassungen über den Formstand des Knöchels des Rechtsaussens bis hin zum Besuch bei der Wahrsagerin alles zu finden ist, lässt sich nur so verstehen. Glauben die Zuschauer an die mühsam zusammengetragenen Zeichen? Ob sie es tun oder nicht ist nebensächlich. Sie schauen zu, weil es ihnen erlaubt, sich vor dem Spiel mit dem Spiel zu beschäftigen. 


\section{Vertrauen als religionssoziologischer Grundbegriff}

An beiden soeben vorgestellten Polen des Kontinuums der Verzauberung von Religion richtete der Blick auf Vertrauen die Aufmerksamkeit auf das religionssoziologisch Interessierende: Die religiöse Erzeugung dauerhaften Selbstmisstrauens und die innerweltlichen Möglichkeiten der Erzeugung von Selbstvertrauen zeigen, wie «Ideen» in der Geschichte wirksam werden» (Weber 1988: 82). Bei ausserweltlichem religiösem Handeln wiederum bewahrt der Blick auf die Praxis des Vertrauens vor intellektualistischen Missverständnissen. Vertrauen sollte deshalb als religionssoziologischer Begriff eine grössere Bedeutung zukommen, als dies zur Zeit der Fall ist.

(1) Dass in der Soziologie Auseinandersetzungen um den Vertrauensbegriff trotz seiner Verwendung weitgehend ausbleiben, ist Zeichen dafür, dass der Begriff selbst keine entscheidenden und dadurch kontroversen Differenzierungen leistet. Tatsächlich wird «Vertrauen» bereits in den meisten Definitionen als diffuses Konzept begründet. So fasst Luhmann (2000 [1968]) Vertrauen «als Mechanismus der Reduktion von Komplexität» und hätte sich damit systemtheoretisch nicht allgemeiner halten können. Oft wird Vertrauen in Graubereichen angesiedelt, so von Simmel (1992 [1908]) als «Hypothese künftigen Verhaltens, als Zustand zwischen Wissen und Nichtwissen». Damit «Vertrauen» nicht zu einer Residualkategorie wird, in die alles gesteckt wird, was durch trennschärfere Begriffe nicht erfasst werden kann, müssen die richtigen Verknüpfungen hergestellt werden, was die besagten Autoren auch durchaus tun: Was ist das Objekt des Vertrauens? Mit welchen Formen von Institutionalisierung hängt Vertrauen zusammen? Wodurch wird Vertrauen bestätigt oder geschwächt? Bedeutungen werden damit über Interaktion, Glaube über Praxis befragt. Über die Fragen nach Objekt, Modus und sozialer Beziehung eröffnet der Vertrauensbegriff der soziologischen Analyse gleichzeitig die semantische Dimension der Bedeutungen und die strukturelle Dimension der möglichen Beziehungen religiöser Kommunikation.

(2) Die Forderung nach einer prominenteren Platzierung des Vertrauensbegriffs muss sich der Frage nach dessen Beziehung zum Begriff von Glauben stellen. Dabei sind die Schwierigkeiten, die sich Religionssoziologie und Religionswissenschaft mit "Glauben» als zentraler Kategorie einhandeln, beträchtlich. Ausgehend von der Annahme, es fände sich in jeder Religion ein Gebäude von Glaubensvorstellungen, wird dazu geschritten, die Symbolsysteme 
zu rekonstruieren, die Glaubenssätze und Gottheiten miteinander in Verbindung zu bringen und zu einem kohärenten Ganzen zu fügen. Gerade von religionswissenschaftlicher Seite wird die Rekonstruktion von Clifford Geertz' «Symbolsystemen» oft als Hauptaufgabe gesehen. Dazu wird ein Reich kultureller Bedeutungen in Form von «Wissen» und «Glauben», von der Ebene sozialer Handlungen und Beziehungen getrennt, erhoben und in Buchform systematisiert. Der Kohärenzzwang wissenschaftlichen Schreibens führt dabei zur Konstruktion einer Systematisierung ohne Entsprechung im Feld. Spätestens seit der Writing CultureDebatte kommen Sozialwissenschaftler zwar nicht darum herum, die Erzeugnisse ihrer Arbeit als Konstruktionen zu identifizieren, zu verhindern ist jedoch zudem, dass solchen etischen Konstruktionen Wirkmächtigkeit auf der emischen Ebene unterstellt wird. Frazers Missverständnisse dürften gerade darauf beruhen. Er systematisierte Mythen und Glaubensvorstellungen und unterstellte sie als Gründe für die Durchführung der ebenfalls beobachteten Rituale. Dies führte $\mathrm{zu}$ einem wissenschaftlich schwer verständlichen Resultat: Hunderte von Stämmen tanzen im Glauben an magisch-meteorologische Gesetzmässigkeiten völlig wirkungslose Regentänze und das während Jahrhunderten. Frazer konnte dies nur erklären, indem er den beobachteten Akteuren unbelehrbare Dummheit in die Schuhe schob. Wittgensteins Zorn überrascht da nicht, denn die Regentänzer handeln nicht entsprechend der ihnen unterstellten, ausdifferenzierten Glaubensvorstellungen, sondern vertrauen auf den Vollzug des Handelns, genauso, wie man nicht an Sprache glaubt, sondern auf das Reden vertraut. Die Logik geht in der Praxis auf und deren Vollzug ist ein für die Beteiligten verlässlicher und auch für den Wissenschaftler beobachtbarer Vorgang. "Glaube» steht zwar im Zentrum zahlreicher soziologischer Religionsdefinitionen, aber nicht aller Religionen.

(3) Damit entfällt der Bedarf für einen Begriff von Glauben keineswegs. Glaubensvorstellungen können sich innerhalb der religiösen Praxis semantisch und strukturell ausdifferenzieren und in Theologien sowohl überdacht als auch verändert werden und wiederum Konsequenzen auf der Handlungsebene haben. Webers Arbeit zum Zusammenhang von asketischem Protestantismus und modernem Kapitalismus bietet ein Beispiel dafür, wie ein systematisierter Korpus religiöser Ideen Interessen formte. Damit ein ausdifferenziertes Gefüge religiöser Ideen zu einem Glauben und einer darauf basierenden, neuen Konfiguration von Vertrauen führen kann, wie sie Weber feststellte, muss eine Trägerschaft dieser Theologie vor- 
handen sein, die auf einen regulierten Zugang zur Gestaltung der Lehre, insbesondere aber auf Strukturen ihrer Verknüpfung mit dem Glauben und Handeln der Laien bauen kann: Glaubensüberzeugen bedürfen, sind sie Teil einer ausgeklügelten Lehre, einer doppelten Kontextualisierung, in der Lehre und im Leben, damit ihnen im Handeln vertraut wird. Die Differenzierung vonVertrauen und Glaube und die dadurch wachsende Distanz zwischen einer Systematik des theologischen Denkens von der religiösen Pragmatik des Alltags muss durch Vermittlungsleistungen wieder aufgefangen werden, soll sich nicht, wie von Weber festgestellt, das diesseitige Vertrauen von seinem Ursprung im religiösen Glauben verselbständigen.

(4) Konzepte einer umfassenden Wertintegration, wie sie bis in die 70er Jahre insbesondere Talcott Parsons verfochten hat, sind in der Soziologie aus der Mode gekommen. Die Frage nach normativer Integration rückte in den Hintergrund, die, dem jeweiligem Ansatz entsprechende Frage nach interaktiven, praktischen oder konfliktiven Vollzügen von auch unordentlichen Ordnungen ins Zentrum des Interesses. Diesen Entwicklungen folgend, sollten Religionssoziologie und Religionswissenschaft ihren Fokus verändern. Die Rekonstruktion von Symbolsystemen sagt noch nichts über ihre soziale Geltung aus und trotz allfälliger «Repräsentativität» ist auch die Abfrage von im Individuum vorhandenen Glaubensvorstellungen soziologisch noch wenig interessant. Dass ihre Diversität hoch, die Verbindlichkeit gering und die individuelle Mobilität beträchtlich ist, braucht nicht durch weitere quantitative Studien mit leicht veränderten Prozentverhältnissen belegt zu werden. Die Frage nach Vertrauen richtet dagegen den Fokus auf die hohe Plausibilität, die Individuen ihren eigenen religiösen Entscheidungen beimessen, auf das Misstrauen gegenüber hoch integrierten Formen religiöser Zugehörigkeit oder auf die beständige Zuwendung zu kirchlichen Ritualen, die keiner Übereinstimmung in Glaubensfragen bedarf. Damit richtet sie den Blick an hochmobilen Gesichtspunktmengen vorbei auf diejenigen Strukturen, die Soziologen an Religionen interessieren.

\section{Literatur}

Durkheim E. (1979): Les formes élémentaires de la vie religieuse. Le système totémique en Australie (Bibliothèque de philosophie contemporaine), Paris: Presses Universitaires de France. 
Frazer JG. (1963): The Golden Bough. A Study in Magic and Religion (St. Martin's Library), London: Macmillan.

Luhmann N. (2000): Vertrauen. Ein Mechanismus der Reduktion sozialer Komplexität. 4. Auflage (UTB für Wissenschaft 2185), Stuttgart: Lucius \& Lucius.

Parsons T. (1964): The Social System, New York:The Free Press.

Simmel G. (1992): Soziologie. Untersuchungen über die Formen der Vergesellschaftung (stw 811; Georg Simmel Gesamtausgabe, Band 11), Frankfurt am Main: Suhrkamp.

Weber M: (1988): Die protestantische Ethik und der Geist des Kapitalismus, In: Weber, Max: Gesammelte Aufsätze zur Religionssoziologie I (UTB für Wissenschaft 1488), Tübingen: J.C.B. Mohr (Paul Siebeck): 17-206.

Wittgenstein L. (1975): Bemerkungen über Frazers «The Golden Bough», In: Wiggershaus R. (Hg.): Sprachanalyse und Soziologie. Die sozialwissenschaftliche Relevanz von Wittgensteins Sprachphilosophie (stw 123), Frankfurt am Main: Suhrkamp: 37-58.

— Dr. des. Rafael Walthert ist Assistent am Religionswissenschaftlichen Seminar der Universität Zürich. 